\title{
Inferring thermal turbulent structures properties in the wake of an array cylindrical obstacles
}

\author{
Saad Mulahasan ${ }^{1, *}$ and Thorsten Stoesser ${ }^{2}$ \\ ${ }^{1}$ Al-Mustensiriyah University, Civil Engineering Department, Baghdad, Iraq \\ ${ }^{2}$ Cardiff University, Cardiff School of Engineering, Hydro-environmental Research Centre, Cardiff, \\ UK
}

\begin{abstract}
In this study a thermal camera was used to inferring the properties of thermal turbulent structures at the water surface of an array of emergent vertical cylindrical obstacles located in staggered fashion at one side of an open channel flow. Three different diameters $(\mathrm{D}=1.25 \mathrm{~cm}, 2.5$ $\mathrm{cm}$ and $5.0 \mathrm{~cm}$ ) were tested. A thermal camera SC640 was used to record a series of snapshots of the water surface temperature distribution in the wake of the cylinders at section where the flow was uniform and fully developed. The selection was made after watching a video to locate the most suitable areas for eddies and turbulence development. For the selected location, temperature values were extracted for each sequence at five selection points in the wake region using Matlab software. Time series of temperature for the snapshots of the five selected points in the area of eddy formation were drawn. The energy of the eddies were extracted using Fourier transforms. The results showed successive peaks with a slope of $5 / 3$. Evaluation of the eddies in the wake of the cylindrical rods showed that the energy of the eddies is small at high frequency and large eddies were observed at low frequencies.
\end{abstract}

\section{Introduction}

With the help of visualization techniques such as digital cameras, remote sensing, laser Doppler anemometer LDA, and non-intrusive flow measurement apparatus such as particle image velocimetry PIV, and thermal cameras IR many unclear aspects of flow phenomena behavior have been explained and deeply studied.

Previous experimental studies used non-intrusive measurements focused mainly on the signature of the thermal structures. For example, a method similar to that of particle image velocimetry was used to tracking large-scale turbulent structures motion from fluctuating thermal patterns of vortices on a heated wall in an open channel flow [1]. The thermal flow patterns were used rather than seeding particles to extract the convective velocity. Time series of the thermal convection velocity in the $\mathrm{x}$ direction is much higher than that in the $\mathrm{z}$ direction. It was observed that the characteristics of the fluid flow can be extracted from the analyzing of the spatial and temporal thermal images. The hydrodynamic in the nearsurface region of a turbulent Cliff-type open channel flow and the characteristics of the

* Corresponding author: irrigation 2010@yahoo.com 
wavy free surface were investigated [2]. FLIR camera is used to visualize the free surface thermal structures. It was shown that these thermal images of the free surface are nonuniform as a result of complexity of wave and turbulence. The IR image shows the "strike" structures in the form of elongated strips of "hotter" and "cooler" liquid. Variation to the data of heat surface distribution with time and then a power spectral density analysis was applied. The spectral analysis shows the distribution of the power contained in a signal. A new method of burst distribution detection based on visual observation of the thermal spots on the free fluid surface was investigated [3]. Infrared camera was able to detect these coherent structures and their frequent appearance on the surface, while in high dragreduction $530 \mathrm{ppm}$ this bursting phenomenon was not observed. Infrared imaging technique was used to extract flow properties such as surface velocity from movement of thermal patterns on the water surface [4]. Geometric properties and velocities associated with micro scale breaking waves using thermal imaging cameras were investigated [5]. Particle image velocimetry (PTV) was used to investigate the characterize portions of the free-surface flow patterns in a wide-open channels with a movable bed at bulk Reynolds numbers between 8500 and 45,500 [6]. Results showed that longitudinal streaks and vertical vortex streets were monitored. Unsteady heat transfer on the wall of a thin conductive film of a turbulent boundary layer was examined using an infrared thermograph technique [7]. Time-spatial distribution of heat transfer coefficient on the wall of a turbulent boundary layer was analyzed by the power spectrum of the temperature fluctuation. Flow properties such as velocity and vorticity were investigated at Reynolds number, $\mathrm{Re}=8000$ for a submerged sphere. IR camera and PIV techniques were used. The wake of the submerged sphere was monitored by IR camera, while PIV was used to extract the dynamics of these coherent structures [8]. Flow characteristics such as velocity evolution, kinetic energy and dissipation at the water surface in a tidal river were investigated. Thermal infrared (IR)based particle image velocimetry PIV in comparison to acoustic velocimetry (ADVs) results were used [9]. Statistical analysis was used to a 5-min ADV and IR-based PIV techniques showed good agreements between these two techniques in calculation of the velocities. Surface flow characteristics in a tidal river were estimated from IR images based PIV delineated boils [10]. Statistical properties of very near to the surface thermal structures exhibit a $-5 / 3$ turbulent cascade. And these boils appealed significant greater velocity variance. Large-scale turbulent structures (boils) of tidal flow over a $60 \mathrm{~m}$ deep sill were investigated with both thermal and visible imagery techniques [11]. To identify and characteristic large scale quasi 2D eddies (velocity and vorticity) at shallow conditions, turbulent mixing process and energy dissipation in rivers and estuaries was numerically investigated. Statistical of turbulence based on remote sensing data and in-situ field data displayed the frequency spectra from the time series of PIV measurements of the surface velocity map monitored by thermal camera [12].

None of these studies, however, investigate the relationship between CS and the turbulent energy of the vortices. The present work being able to monitor the thermal coherent structure and then to characterize at some extent the turbulent structure of the flow through the time series of temperature measured with a thermal camera. The most suitable place of eddies in the wake region was chosen after watching recorded video of each rod size experiment at different locations. The ideal location is in the interface between the undisturbed cold water and the warm water flow as shown in the fourth square in Fig.1. The zone should be close enough to the seeding (hot water) point so that the mixing is not too high and the temperature gradients too dissipated, but also far enough so that the eddies have space to develop and have passed through some rods already.

The purpose of this study is to visualize and educe coherent flow structures in the flow through arrays of emergent rods placed on a floodplain of a compound channel. 


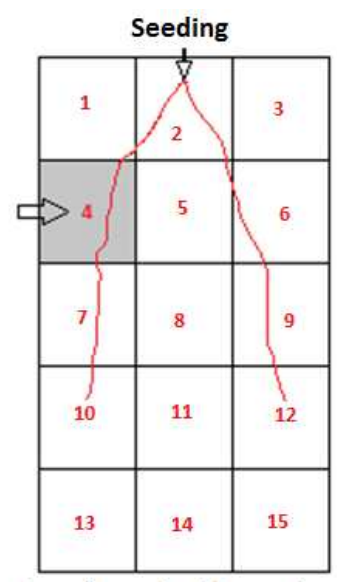

Fig.1. Selection the suitable region of the wake.

\section{Laboratory Experiments}

Laboratory experiments were carried out in a $10 \mathrm{~m} \mathrm{X} 1.20 \mathrm{~m} \mathrm{X} 0.3 \mathrm{~m}$ recirculating tilting flume at Cardiff University, UK. A compound channel cross section with one floodplain with shallow flow conditions was performed by installing plastic sheets along one side of the flume, $76 \mathrm{~cm}$ wide, $24 \mathrm{~mm}$ thickness and channel side slope of $1(\mathrm{H}): 1(\mathrm{~V})$. Circular wooden rods with different diameters; $\mathrm{D}=1.25 \mathrm{~cm}, 2.5 \mathrm{~cm}$ and $5.0 \mathrm{~cm}$ were used to mimic rigid emergent vegetation elements. The floodplain was designed as a wholly vegetated floodplain in which the longitudinal and transverse center to center spacing was $12.5 \mathrm{~cm}$. A tailgate that was located at the downstream end of the flume's working section was used to control flow depths. The bed slope of the flume was set to $1 / 1000$. The test section located $7.6 \mathrm{~m}$ down the upstream edge of the flume's working section that was characterized as a fully developed flow condition. A point gauge was used to measure water depths with an accuracy of 1/10 and Nixon Probe Velocimetry was used to measure point velocities. A flow meter was used to measure flow rates and calibrated by velocity measurements and by volumetric flow rate technique. A FLIR Systems Thermal CAM SC640 was mounted vertically above the water surface at a height of $65 \mathrm{~cm}$ at the interface between the main channel and the floodplain at section where a fully developed flow conditions had achieved to record the temperature distribution of the water surface at a rate of 5 frames per second. The SC640 camera has a thermal sensitivity of $30 \mathrm{mK}$ at $30^{\circ} \mathrm{C}$, and its resolution is 640 pixels X 480 pixels. A hot water source is injected continuously from a tank into the flume's working section on the water surface at the main channel/floodplain interface at $7.6 \mathrm{~m}$ of fully developed flow section. Recorded images are stored to a high-density SD-card inside the thermal camera. Multiple flow rates were tested and only flow rate of $11.03 \mathrm{l} / \mathrm{sec}$ was reported in this paper as explained in Table 1. In this table calculation of the Reynolds number is based on the bulk velocity, $U$ and the cylinder diameter, $\operatorname{Re}_{\mathrm{D}}=\mathrm{UD} / v$. Recorded videos were achieved in such a way that the area to be captured is in a part of the entire shear layer area, which consists of 15 squares, five in the longitudinal direction and three squares in the transverse direction, which were checked before the actual runs (see Figure 1). Thermal patterns were clearly observed on the water surface directly on the screen of the thermal camera [13]. The seed water (hot water) mixed rapidly with the flume water in the stream-wise and transverse directions. 
Table 1. Flow conditions.

\begin{tabular}{|c|c|c|c|c|c|}
\hline \multirow[t]{2}{*}{ Case study } & \multirow{2}{*}{$\begin{array}{c}\text { Discharge, } \\
\text { Q (1/sec) }\end{array}$} & Water depth,(cm) & \multirow{2}{*}{$\begin{array}{c}\text { Bulk velocity, } \\
\mathrm{U}(\mathrm{cm} / \mathrm{sec})\end{array}$} & \multirow{2}{*}{$\begin{array}{c}\text { Reynolds } \\
\text { number }\end{array}$} & \multirow{2}{*}{$\begin{array}{l}\text { Froude } \\
\text { number }\end{array}$} \\
\hline & & $\mathrm{H}_{\mathrm{mc}}$ & & & \\
\hline $\begin{array}{l}\text { Fully vegetated, } \\
\mathrm{D}=1.25 \mathrm{~cm}\end{array}$ & 11.03 & 5.38 & 14.73 & 1831 & 0.17 \\
\hline $\begin{array}{l}\text { Fully vegetated, } \\
D=2.5 \mathrm{~cm}\end{array}$ & 11.03 & 5.55 & 14.34 & 3564 & 0.16 \\
\hline $\begin{array}{l}\text { Fully vegetated, } \\
D=5.0 \mathrm{~cm}\end{array}$ & 11.03 & 7.95 & 14.34 & 7128 & 0.16 \\
\hline
\end{tabular}

\section{Results and discussions}

The three measurements considered in Table 1 are characterized by turbulent subcritical flows and Reynolds number are in the range of $\mathrm{Re}=1831 \sim 7128$ and of the bulk flow velocity was in the range $U=14.73-14.34(\mathrm{~cm} / \mathrm{s})$ respectively. These three cases are characterized by the diameter of the rods set as obstacles: Diameter sizes are $1.25 \mathrm{~cm}, 2.5 \mathrm{~cm}$ and $5 \mathrm{~cm}$. The objective is to characterize the developing eddies; hence the preferred places are in the wake of the rods. The selection was made after watching a video to locate the most suitable areas for eddies and turbulence development. Figure 1 shows the area in which the points were selected for three of the data sets but is useful to illustrate the general procedure. The zone should be close enough to the seeding point so that the mixing is not too high and the temperature gradients too dissipated, but also far enough so that the eddies have space to develop and have passed through some rods. The ideal location is in the interface between the undisturbed cold water and the warm water flow (red line). For every case, the following data are provided: i) Points' locations sketch. ii) Time series of Temperature. iii) Spectral analysis of turbulence based on the aforementioned time series. At the end of recording video files, the next step is to export these sequences from the thermal camera into the FLIR software first, and then transferred them from the FLIR software to Matlab files. For every test a total number of 187 snapshots of the instantaneous temperatures were achieved at a rate of 5 frames per second. The average temperature calculation from series of sequences of 187 images ( 640 X 480 pixels) is carried out for each square. The thermal patterns created by the coherent structures of turbulent channel flow are considered. The results of the eddies evolution in the wake of the rods for the wholly vegetated floodplain tests were monitored and explained by the temperature distribution as shown in Figures 2(a, b, and c) for the rod sizes $1.25 \mathrm{~cm}, 2.5 \mathrm{~cm}$ and $5.0 \mathrm{~cm}$ respectively. In these Figures it was shown that as vegetation elements densities (area of vegetation elements /area of the bed) are increased the eddies past the rods are inhibited (see Fig.2a). In contrast, active eddies were observed in both cases of sparse and medium densities of vegetation elements (Figures 2a, b). In Figures $2 \mathrm{a}, \mathrm{b}$ and $\mathrm{c}$ the flow is from the bottom to the top of the images and the coordinates of Figure 2 are in pixels.

For high image resolution, temperature values were extracted for each sequence at five selected points ( 1 to 5 ) that were selected subjectively in the wake region using Matlab software. Figures $3(\mathrm{a}, \mathrm{b}$ and $\mathrm{c}$ ) are showing the variation of the temperature distribution versus time at the selected points in the wake of the cylinders within a fully developed flow section based on 187 sequences measurements. Along the time series frames (37.4 seconds) successive peaks are appealed for all three cylinders sizes. The power spectral analysis was applied. The results showed the successive peaks were observed with a slope of $-5 / 3$ and insured an indication of turbulent flows first (see Fig.4). The energy contained in a single represents the energy power of the eddies over the frequency $1 / T$. Figures $4 a, b$ and $c$ 
showed that the energy of the eddies is small at high frequency, while for low frequencies there are large eddies.

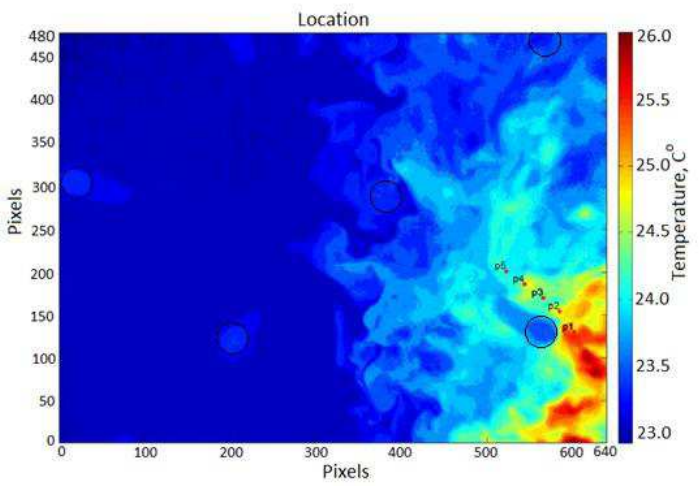

a)

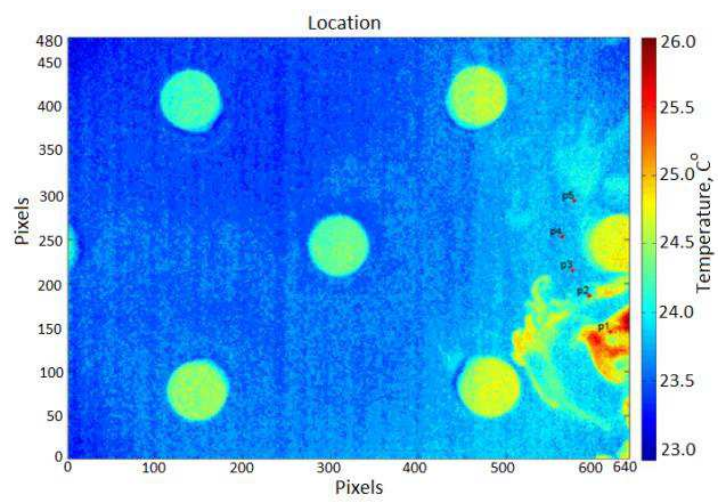

b)

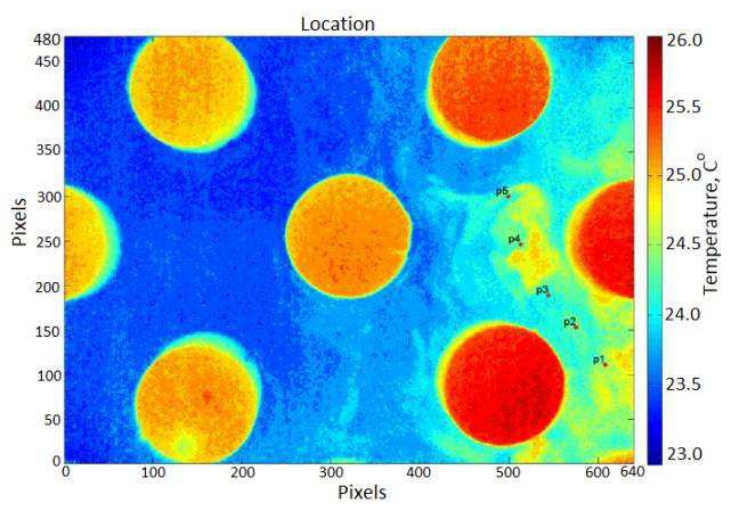

c)

Fig.2. Eddies evolution in the wake of the rods for the wholly vegetated floodplain with rod diameter a) $1.25 \mathrm{~cm}, \mathrm{~b}) 2.5 \mathrm{~cm}$ and c) $5.0 \mathrm{~cm}$. (Flow is from the bottom to the top of the images). 


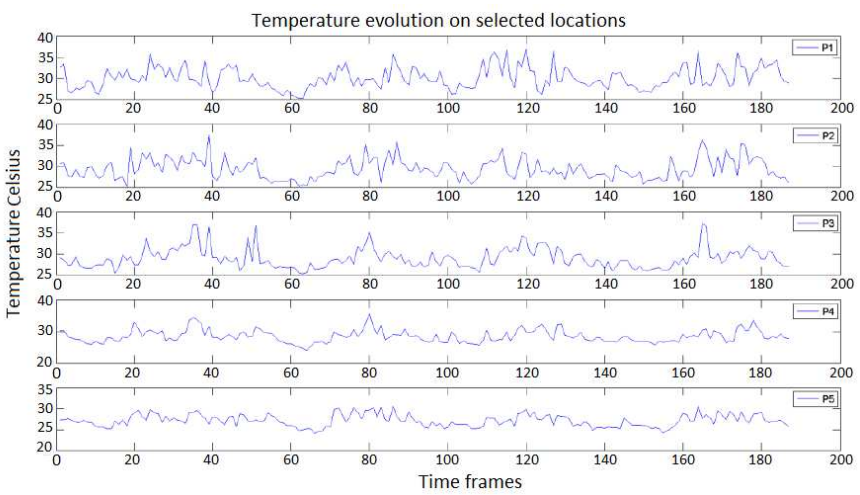

a)

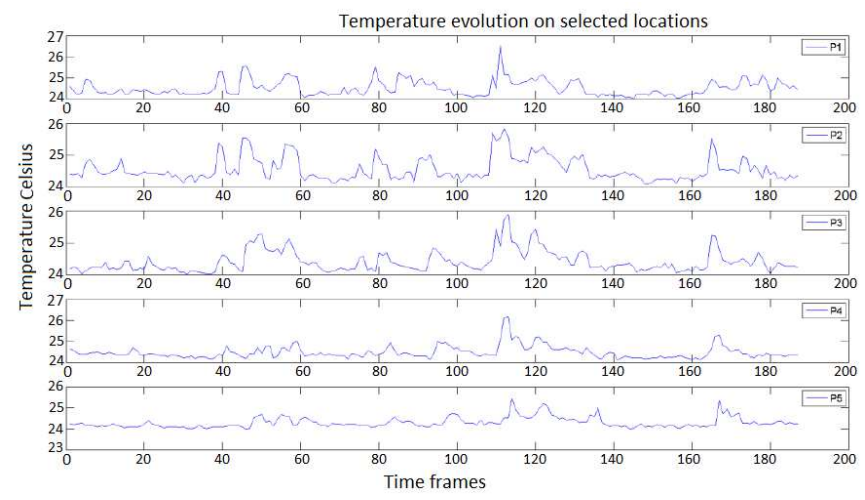

b)

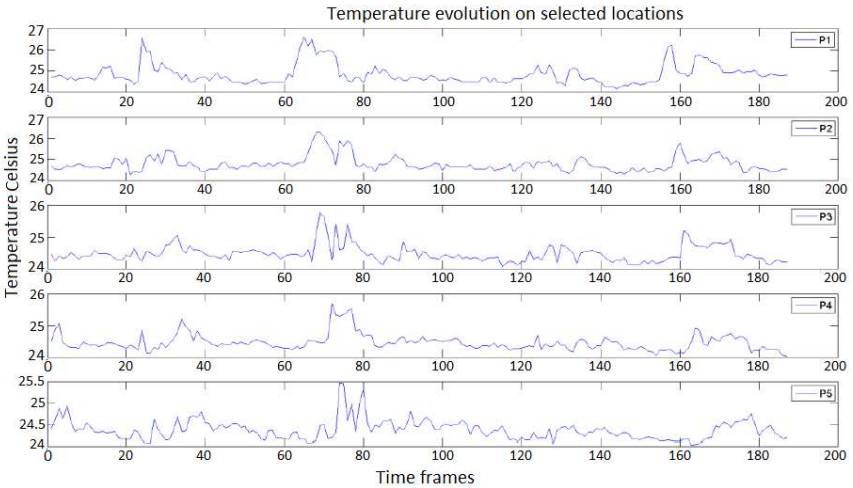

c)

Fig.3. Time series data of the temperature at five selected points (1 to 5) in the wake of the cylinders within a fully developed flow section distributed in a staggered arrangements on the floodplain with rod diameter of a) $1.25 \mathrm{~cm}, \mathrm{~b}) 2.5 \mathrm{~cm}$ and c) $5.0 \mathrm{~cm}$. Data of the time variation of the temperature was based on 187 sequences measurements. 


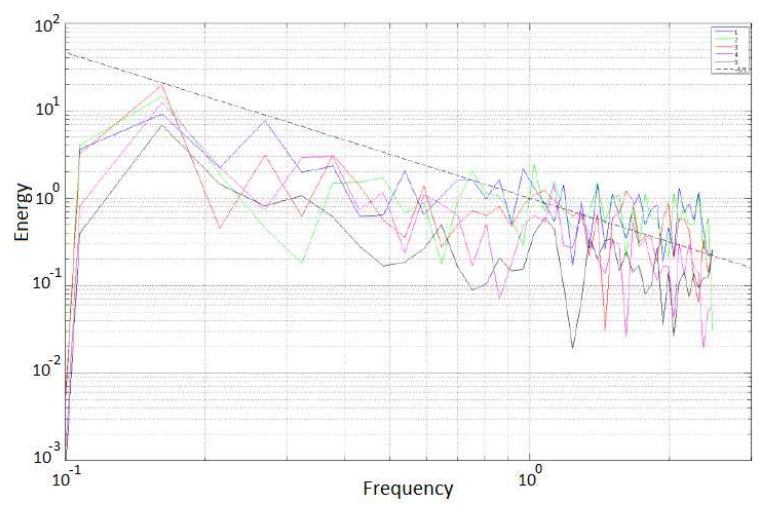

a)

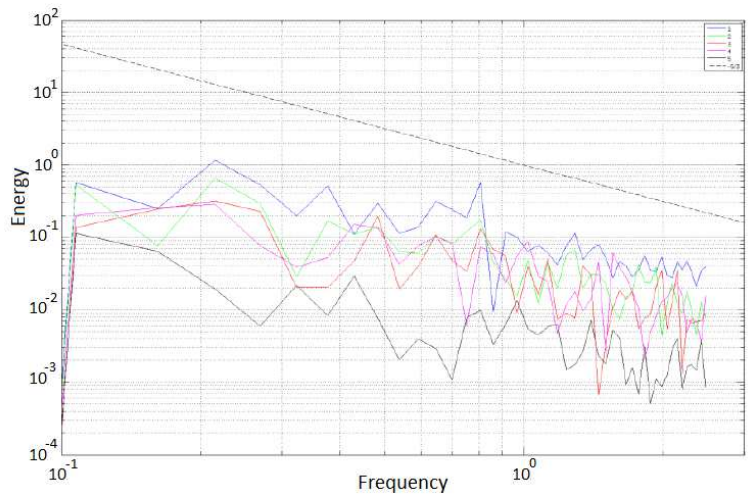

b)

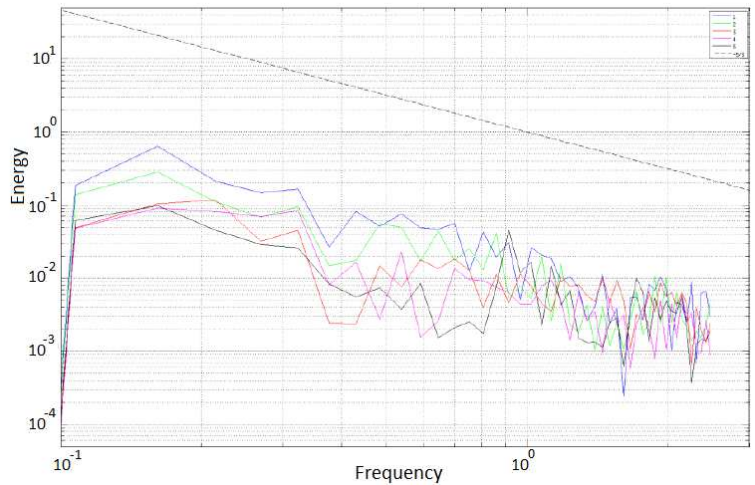

c)

Fig.4. Fourier transforms for the five selected points (1 to 5) in the wake of the rods for the case of the wholly vegetated floodplain of rod diameter a) $1.25 \mathrm{~cm}, \mathrm{~b}) 2.5 \mathrm{~cm}$ and c) $5.0 \mathrm{~cm} . \mathrm{D}=5.0 \mathrm{~cm}$. 


\section{Conclusions}

A thermal system was used to visualize and educe coherent flow structures in the flow through arrays of emergent rods placed on a floodplain of a compound channel. Active eddies in both cases of sparse and medium densities of vegetation elements were observed, while the eddies past the largest rods sizes are inhibited. Time series of five selected points in the wake of the cylinders showed successive peaks. The power spectral analysis insured an indication of turbulent flows and the energy of the eddies appealed small at high frequency, while for low frequencies there are large eddies.

\section{Acknowledgments}

The authors would like to thank Iraqi government for its finance support, and the technical staff of the hydraulic laboratory, school of engineering at Cardiff University, UK for their efforts in instrumentation installation.

\section{References}

1. G. Hetsroni, B. Hu, T. A. Kowalewski, A. Mosyak, Experiments in Fluids 30, 286-294 (2001)

2. S. Smolentsev, B. Freeze, N. Morley, M.Abdou, Fusion Engineering and Design 63-64, 397-403 (2002)

3. A. Mosyak, G. Hetsroni, Experiments in Fluids 37, 40-46 DOI 10.1007/s00348-0040782-6 (2004)

4. X. Zhang, and S. Harrison, Physics of Fluids, 16(1), L5-L8 (2004).

5. A. T. Jessup and K. R. Phadnis, Meas. Sci. Technol., vol. 16, no. 10, pp. 1961- 1969, Oct. (2005)

6. A. Tamburrino and J. S. Gulliver, Water Resources Research, vol. 43, w11410, doi:10.1029/2007wr005988 (2007)

7. H. Nakamura, Transactions of the Japan Society of Mechanical Engineering Series B, vol. 73 issue 733, pages 1906-1914 (2007)

8. S. I. Voropayev, H. J. S. Fernando and C. Nath, Journal of Visualization, Vol. 12, No. 4, 285 (2009)

9. C. C. Chickadel, S. A. Talke, A. R. Horner-Devine, and A. T. IEEE Geoscience and remote sensing letters, vol. 8, no. 5, september (2011)

10. S. A. Talke, A.R. Horner-Devine, C.C. Chickadel, and A. T. Jessup, submitted to Journal of Geophysical Research, vol. 118, 6965-6981 (2013)

11. G. O. Marmorino *, G. B. Smith and W. D. Miller, Remote Sens., 5, 3239-3258; doi: $10.3390 /$ rs5073239 (2013)

12. S. A. Talke, Ed Zaron, and C. C. Chickadel, Report, Portland State University, Civil and Environmental Engineering Department,PO Box 751-CEE,Portland,OR,972070751 Sep. (2013)

13. S. Mulahasan*, T. Stoesser and F. Alvarez, 10th Pacific Symposium on Flow Visualization and Image Processing Naples, Italy, 15-18 June (2015) 